\title{
Abnehmen kann nur, wer satt und zufrieden ist
}

Von 4575 Menschen mit Typ-2-Diabetes zeigen 95\% abdominelle Adipositas. Die Phase ,gestörte Glukosetoleranz' findet etwa 4-7 Jahre vor Diagnose des Typ-2Diabetes statt. Dann ist die Betazell-Funktion um 50\% reduziert. Nach Diagnose sinkt sie weiter um $6 \%$ pro Jahr.

Ist Diabetes „frisch diagnostiziert“, ist Remission zu 12\% umsetzbar. Würde man schon den Prädiabetes „therapieren“, stiegen die Heilungsraten erneut. Nach Dr. med. Mattias Riedl, Hamburg, bietet allein der AXA-Versicherungskonzern DMP's dafür.

Eine kurze Krankheitsdauer und mögliche Senkung des BMI sind Erfolgsfaktoren für die Remission, genauso wie höheres Alter. Der ideale BMI beträgt $22,5-25 \mathrm{~kg} /$ $\mathrm{m}^{2}$. Zum einen ist Übergewicht der größte Killer (pro $5 \mathrm{~kg} / \mathrm{m}^{2}$ Steigerung steigt die Gesamt-Sterblichkeit um ca. 30\%), zum anderen gelten ca. 25\% der Adipösen als sog. „happy obese“, da sie nicht zu Stoffwechselerkrankungen neigen.
Riedl benötigt eine stadiengerechte Therapie, weg vom BMI-Denken hin zum kanadischen Edmonton Obesity Staging System (EOSS, Sharma AM \& Kushner RF, Int J Obes 2009). Zunächst fragt man: Bereitet Ihnen Ihr Gewicht Sorge? Sagt der Patient „Nein“, ist das Thema beendet. Sagt er "Ja“ erkundet man seine Motivation per Skala.

Realistisches Ziel ist die Abnahme von 0,5-1 kg pro Woche. Das Vorsetzen von Regeln einer Diät bringt nichts. Besser ist es, sich anzuhören, welche Ernährungsgewohnheiten der Patient hat und dann „schlechte“ herauszupicken und möglichst vergessen zu machen. Ist ein Patient nicht bereit, eine Gewohnheit zu ändern, lässt man sie so und kümmert sich um die nächste „schlechte“ und das funktioniert nur in Einzeltherapie.

Abnehmen kann nur, wer satt und zufrieden ist. Am wenigsten zufrieden macht eiweißarme Kost + hoher glykämischer Index (Abbruchrate 37\%). Am zufriedensten macht eiweißreiche Kost + niedriger glykämischer Index (Abbruchrate $27 \%$ ).

Eva L., 56 Jahre, $\mathrm{HbA}_{1 \mathrm{c}} 8,5 \%$, hat einen BMI von 37. Sie erhielt ein Inkretinmimetikum + Metformin + Detemir als BOT (120 Einheiten). Der Diabetologe entfernt das Inkretinmimetikum und ergänzt ein Bolusinsulin und darunter nahm sie $20 \mathrm{~kg}$ zu. Riedl setzte das Bolusinsulin ab, glich die erhöhten Tageswerte mit dem länger wirksamen Insulin (Abasarglar ${ }^{\circledR}$ ) aus und setzte das Inkretinmimetikum wieder ein. Ihr Ergebnis: Gewichtsreduktion um $18 \mathrm{~kg}$ in 6 Monaten, Dosisreduktion des Basalinsulins um 30\% und die Patientin fühlt sich aktiver.

Nana Mosler, Leipzig

Quelle: Workshop „Paradigmenwechsel beim Insulinstart: Ernährungsmanagement bremst Gewichtszunahme" anlässlich des Diabetes Kongress, am 5. Mai 2016 in Berlin. Veranstalter: Lilly Deutschland $\mathrm{GmbH}$,

Boehringer Ingelheim Pharma GmbH \& Co. KG

\section{Mehr Sicherheit bei Diabetes-Kontrolle mit eHealth-Lösungen}

Das Management des Diabetes mellitus befindet sich dank der Möglichkeiten, die die digitale Welt mittlerweile bietet, seit Jahren in einem grundlegenden Wandel. Welchen Stand eHealth-Lösungen heute bereits erreicht haben, zeigte sich auf einer Veranstaltung anlässlich der 9. ATTD (Advanced Technologies \& Treatments for Diabetes)-Tagung in Mailand. Von dem Fortschritt profitieren beispielsweise Patienten mit einem Typ-1-Diabetes, die tagtäglich mehrmals ihren Blutzucker bestimmen müssen, um dann die benötigte Menge an Insulin zu bestimmen. Diese Bolusrechnung ist ein komplexer Vorgang, bei dem Aspekte wie geplante Speisen, Sport, Alkohol oder Stress mit berücksichtigt werden müssen, um kurzfristig Hypoglykämien und langfristig schwere Komplikationen zu vermeiden. Ein automatischer Bolusrechner wie der Accu Chek Bolusrechner bietet hier einen erheblichen Zusatznutzen, wie Dr. Kirsten Nørgaard [1] von der Universitätsklinik Hvidovre,
Dänemark, anhand der Ergebnisse der Steno-ABC-Studie zeigen konnte. An dieser Studie über 12 Monate nahmen langjährige Typ-1-Diabetiker mit einem $\mathrm{HbA}_{1 \mathrm{c}}$ um die $9 \%$ teil. Eine Gruppe war in der Methode der fortgeschrittenen Kohlenhydratmessung (advanced carbohydrate counting), die andere im Umgang mit dem automatischen Bolusrechner geschult. Nach einem Jahr war das $\mathrm{HbA}_{1 \mathrm{c}}$ in der einen Gruppe um 0,2\%, unter Einsatz des Gerätes aber um 0,5\% abgefallen. Wie Nørgaard beobachten konnte, beurteilten die Patienten den Accu-Chek Bolusrechner als nützlich, benutzerfreundlich und als große Hilfe.

Die Kontakte zwischen Patient und medizinischen Betreuern bezüglich der Kontrolle und Überwachung der Blutzuckereinstellung abseits der Arztbesuche laufen heute vielfach noch über E-Mails, Telefon oder Fax ab. Um beide Seiten zusammenzubringen und fundierte Therapieentscheidungen treffen zu können, bezeichnete Prof. Concetta Irace, Universität von Catanzaro Magna Græcia, Italien, das Accu Chek ${ }^{\circledR}$ Connect System als bessere Option. Das System besteht aus dem Accu Chek Aviva Connect Blutzuckermesssystem, einer App und einem webbasierten Portal. Wie Irace erklärte, erlaubt es eine schnellere und zuverlässigere Auswertung der Blutzuckerwerte als bisher. Als Belege führte Irace die ACCRUES (Accu Chek Connect Reports Utility and Efficiency Study) und die Accu Chek Friends Connect Study an. Die Studienteilnehmer stimmten überwiegend darin überein, dass sich Blutzuckerverläufe mit dem innovativen System leichter erfassen und besser verstehen lassen. Die Patienten fühlen sich sicherer in der Kontrolle des Stoffwechsels und müssen weniger Hypoglykämien fürchten.

Martin Bischoff, Planegg

Quelle: Medienevent „The Interconnected Perspective: Integrated PDM \& eHealth go hand in hand for improved outcomes" anlässlich des 9. ATTD-Kongresses am 3. Februar 2016 in Mailand. Veranstalter: Roche Diabetes Care 